\title{
Clinical Manifestations in Intoxications by Tricyclic Antidepressants
}

\author{
A Fernandez Ibanez*1, R Ugalde Herra ${ }^{2}$, JA Rodriguez Getino ${ }^{1}$, JB Garcia Casas ${ }^{1}$ and JC Diaz Suarez ${ }^{1}$ \\ ${ }^{1}$ Department of Medicine, University of Oviedo, Spain \\ ${ }^{2}$ Hospital Universitario Central de Asturias, Oviedo, Spain
}

Received: 制 August 23, 2018; Published: 㭗 August 29, 2018

*Corresponding author: A Fernandez Ibanez, Department of Medicine, University of Oviedo, Spain

\begin{abstract}
Introduction: Tricyclic antidepressants are used in multiple psychiatric disorders such as depressive syndrome or attention deficit hyperactivity disorder. Acute intoxications by this drug if not treated in time, have a high mortality due to the cardiovascular effects that occur in the body.

Objective: Detect acute poisoning by tricyclic antidepressants with the analytical values in serum of patients who have symptoms compatible to establish treatment early.

Method: Review of the clinical history of patients who in the urine drug screening test were positive for tricyclic antidepressants and had a compatible electrocardiogram. Venous blood samples were processed in Cobas 6000 e501 (Roche Dignostics) by the homogeneous enzyme immunoassay technique.
\end{abstract}

Results: Differentiation in 3 groups of patients according to the plasma concentration values.

Conclusions: The quantification of the plasma concentration of tricyclic antidepressants offers additional information to confirm the diagnosis but not for the urgent treatment of these intoxicates because the free concentration of the drug is modified with $\mathrm{pH}$ or the individual variability.

It must remember that many of these intoxications are associated with benzodiazepine poisoning, so treatment with flumazenil is contraindicated because it can produce convulsions.

\section{Introduction}

Tricyclic antidepressants are used in multiple psychiatric Disorders such as depressive syndrome or attention deficit hyperactivity disorder. Since 90's the prescription and poisoning by these drugs has decreased significantly by the introduction of selective serotonin reuptake inhibitors. Intoxication due to this type of substances occurs in school patients below 5 years old, who ingest them accidentally at home or adolescents and adults who take these drugs for their pathology and present high suicidal risk. The pharmacological group of antidepressants constitutes the second most common cause of poisoning, and within this group are the tricyclic antidepressants (TCAs) those with the highest morbidity and mortality because they can produce important cardiovascular and neurological toxicity [1-4]. The effects of TCAs are very varied and can be divided into 3 groups according to the stimulated receptors blocked:

a. Neurotransmitter reuptake inhibitors (norepinephrine, serotonin and dopamine).

b. Blockade of receptors: adrenergic (alpha 1), dopaminergic, histaminergic and muscarinic. c. Blockade of fast sodium channels and late rectifiers of potassium.

Usually, the peak serum concentration is reached between 2 to 8 hours after the administration of the drug. However, in an overdose, the bioavailability of the TCAs may vary due to the anticholinergic effect of these substances or with the gastric emptying [5-9]. Inmost cases of TCAs poisoning, the clinical picture appears rapidly, and most deaths occur in the first hours. The TCAs have a high lip solubility, they cross the blood-brain barrier and also, they have a high volume of distribution $(10-20 \mathrm{~L} / \mathrm{kg})$. In some tissues the concentration of the drug is 10-100 times the blood concentration. These characteristics explain why measures such as forced diuresis or hemodialysis are ineffective for the treatment of this type of intoxication

\section{Patients and Methodology}

The criteria for the inclusion of patients were presented neurological symptoms or cardiovascular signs in the electrocardiogram with the urine drug screening test were positive for tricyclic antidepressants and whose laboratory tests requested the determination of TCAs in serum. In the selected period for the 
development of work (2015), 13 acute overdoses was followed up in 29 patients with chronic treatment. Samples were collected from 15 women and 14 men, aged between 22 and 72 years. The venous blood samples were obtained in vacuum tubes with lithium heparin anticoagulant. They were centrifuged at 10,000 rpm, for 5-7 minutes and separated for be processed immediately. The tricyclic antidepressants were analyzed in Cobas 6000 e501 (Roche Dignostics) by the homogeneous enzyme immunoassay technique. The analysis is since the drug present in the sample and the drug marked with the enzyme glucose-6-phosphate dehydrogenase (G6FDH) compete for the conjugation sites of the antibodies [1016].

The activity of the enzyme decreases when conjugated with the antibody so that the concentration of drug in the sample can be measured based on the enzymatic activity. The active enzyme converts oxidized nicotinamide-adenine-dinucleotide (NAD) to $\mathrm{NADH}$, which causes a change in absorbance that can be measured spectrophotometrically. Endogenous serum G6FDH does not interfere because the coenzyme works only with the bacterial enzyme (from Leuconostor mesenteroides) used in the analysis. Keep in mind that the analysis detects the presence of a class of tricyclic antidepressants and their metabolites. Any positive result must be confirmed with the reference method. The response to the analysis of an unknown sample containing a tricyclic antidepressant is a cumulative result of the tricyclic antidepressants and their metabolites with similar structures present in the sample [16-22].

\section{Results}

For the determination of this drug, fasting is recommended 12 hours prior to taking the sample but if it is an overdose this criterion is eliminated because clinicians need to know the value of tricyclic antidepressants. As the interpretation of the results depends on the value of the drug with its reference limit indicating toxicity $(200 \mathrm{mg} / \mathrm{mL})$, and visible symptoms, because of that the patients have been divided into 3 groups.

a) First group (n=5): Patients with mild neurological symptoms who presented disorientation or agitation, hypertension and prolongation of the interval QRS in the electrocardiogram. Plasma concentrations between $(200-300 \mathrm{mg} / \mathrm{mL})$. The management was monitoring of vital signs and electrocardiographic monitoring continuous due to cardiovascular complications associated and establish the protection of definitive airway if there is marked alteration of consciousness.

b) Second group $(n=4)$ : Plasma concentrations between $300-400 \mathrm{ng} / \mathrm{mL}$ whose patients presented cardiac signs of moderate intoxication with gasometry values of $\mathrm{pH}$ between 6,8 and 7,1 (acidemia) and hypotension. The treatment was based in decontamination measures that consisted in a gastric lavage with activated charcoal in the patients whose overdose was maximum one hour ago.

c) Third group $(n=4)$ : These patients have a very severe clinical presentation with cardiac arrhythmias or conduction disorders that are the main cause of mortality, due to the plasma concentrations of these drugs $>400 \mathrm{ng} / \mathrm{mL}$. The treatment was a combination of pharmacological measures included sodium bicarbonate, hypertonic solution, lidocaine and magnesium sulfate if the patient presented torsade de points in the electrocardiogram.

For the correct interpretation of the results, the high therapeutic concentrations, that is, between 200 and $300 \mathrm{ng} / \mathrm{mL}$ and the toxic concentrations of chlorpromazine can give a positive result in the analysis. Therapeutic concentrations of cyclobenzaprine (20 to $34 \mathrm{mg} / \mathrm{mL}$ ) should not give positive results with this test, but higher concentrations of the compound (> $200 \mathrm{ng} / \mathrm{mL}$ ) can show a positive result with the test. The assay also detects thioridazine at therapeutic concentrations $(>1.5 \mu \mathrm{g} / \mathrm{mL})$, diphenhydramine at toxic concentrations ( $>12 \mu \mathrm{g} / \mathrm{mL}$ ), orphenadrine citrate at lethal concentrations (> $6 \mu \mathrm{g} / \mathrm{mL}$ ) and cyproheptadine at therapeutic concentrations $(>0.42 \mu \mathrm{g} / \mathrm{mL})$.

\section{Discussion}

Early recognition of tricyclic antidepressants overdose is essential to reduce the morbidity and mortality of these patients; however, this recognition can be difficult because the early findings in this type of intoxication are not specific. Therefore, the first diagnostic tool in the emergency department should be to prepare a good survey, so that the information obtained can be suspected; it is also important to know the patient's history. Symptoms usually start 30 to 40 minutes after intake, but may be later because of the delay in the gastrointestinal emptying secondary to the anticholinergic effect. The initial clinical picture is characterized by alteration of the consciousness, which will vary from agitation and delirium to drowsiness, stupor or coma, initially there may be hypertension, but also normo or hypotension secondary to blockage of alpha 1 adrenergic receptor, a condition that may evolve a refractory shock. A characteristic manifestation is sinus tachycardia stable that can occur in up to $85 \%$ of patients, this finding, although very frequent, does not usually require treatment and can last up to 7 days after the poisoning.

It's necessary to be considered differential diagnoses such as intoxications by anticholinergics and antihistamines, poisoning by other medications for psychiatric use (phenothiazines: chlorpromazine, promazine) usually the treatment has the combinations of this drugs with TCAs and poisoning by antiarrhythmics mainly of group I3 because produces tachycardias and hypotension. Usually measuring the concentration of TCAs in blood is difficult, because very low values may be present in very serious patients, this depends on the moment of the taking, the amount ingested of the antidepressant and the application of decontamination measures. Multiple studies conclude that TCAs are not dialysable and in general, extracorporeal treatment is not recommended in an intoxication by tricyclic antidepressants because they are small molecules (between 200 and $400 \mathrm{Da}$ ) and therefore can pass through any hemofilter or hemodialyzer, so they can generate "bounce" effects with no clinical benefits in the body. 


\section{Conclusion}

Tricyclic antidepressants produce an inhibitory effect on fast sodium channels, slowing phase 0 of depolarization of His-bundle, Purkinje and myocardial fibers ventricular, this effect results in a prolongation of the interval QRS on the electrocardiogram; this fact has been proposed as one of the mechanisms by which intoxication by TCAs can produce arrhythmias, because this effect facilitates the development of reentries secondary to an unequal delay in driving. TCAs have a narrow therapeutic margin and have been documented serious complications with relatively low doses. In general, it is considered that doses higher than $5 \mathrm{mg} / \mathrm{kg}$ are doses toxic, and doses between $10-20 \mathrm{mg} / \mathrm{kg}$ can lead to toxicity serious. Lethal cases with higher doses have been reported of $25 \mathrm{mg} / \mathrm{kg}$.

It's important to determine the levels in blood of ADT, can be useful to confirm the diagnosis in a patient with a compatible clinical picture and not clear etiology. Although it should be considered that the test has false positives when the patient has taken: carbamazepine, diphenhydramine, phenothiazines and phenytoin, which it would limit the interpretation of this test. Other examinations that must be requested depend on the context of the patient, if there is an alteration of consciousness without a clear cause, electrolytes, blood glucose, kidney function, transaminases, thyroid hormones, skull CT and gasometry, in this way the possibility of differential diagnoses. It must remember that many of these intoxications are associated with benzodiazepine poisoning, so treatment with flumazenil is contraindicated because it can produce convulsions.

\section{References}

1. Mc Kenzie MS, McFarland BH (2007) Trends in antidepressant overdoses. Pharmacoepidemiol Drug Saf 16(5): 513-523.

2. Body R, Bartram T, Azam F (2011) Guidelines in Emergency Medicine Network (GEMNet): guideline for the management of tricyclic antidepressant overdose. Emerg Med J 28(4): 347-368.

3. Paksu S, Duran L, Altuntas M (2014) Amitriptyline overdoes in emergency department of university hospital: evaluation of 250 patients. Hum Exp Toxicol 33(9): 980-990.

4. Bailey B, Buckley NA, Amre DK (2004) A meta-analysis of prognostic indicators to predit seizures, arrhythmias, or death after tricyclic antidepressant overdose. J Toxicol Clin Toxicol 42(6): 877-888.

5. Buckley NA, Chevalier S, Leditschke IA, O'Connell DL, Leitch J, et al. (2003) The limited utility of electrocardiography variables used to predict arrhythmia in psychotropic drug overdose. Crit Care 7(5): R101107.

ISSN: 2574-1241

DOI: $10.26717 /$ BJSTR.2018.08.001659

A Fernandez Ibanez. Biomed J Sci \& Tech Res

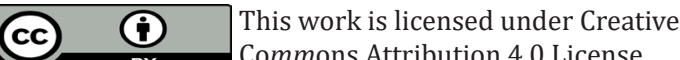

Submission Link: https://biomedres.us/submit-manuscript.php
6. Niemann JT, Bessen HA, Rothstein RJ, Laks MM (1986) Electrocardiographic criteria for tricyclic antidepressant cardiotoxicity. Am J Cardiol 57(13): 1154-1159.

7. Thanacoody HK, Thomas SH (2005) Tricyclic antidepressant poisoning. Toxicol Rev 24(3): 205-214.

8. Liebelt EL, Ulrich A, Francis PD (1997) Serial electrocardiogram changes in acute tricyclic antidepressant overdoses. Crit Care Med 25(10): 17211726.

9. Callaham M (1979) Tricyclic antidepressant overdose. J Am Coll Emerg Physicians 8(10): 413-425.

10. Kerr GW, Mc Guffie AC, Wilkie S (2001) Tricyclic antidepressant overdose: a review. Emerg Med J 18(4): 236-241.

11. Callaham M, Kassel D, Cruz S (1985) Epidemiology of fatal tricyclic antidepressant ingestion: implications for management. Ann Emerg Med 14(1): 1-9.

12. Blackman K, Brown S, Wilkes G (2001) Plasma alkalinisation for tricyclic antidepressant toxicity: A systematic review. Emerg Med 13(2): 204 210 .

13. Veris van Dieren J, Valk L, van Geijlswijk I, Tjan D, van Zanten A (2007) Coma with ECG abnormalities: consider tricyclic antidepressant intoxication. Neth J Med 65(4): 142-146.

14. Hoffman JR, Votey SR, Bayer M, Silver L (1993) Effect of hypertonic sodium bicarbonate in the treatment of moderate-to-severe cyclic antidepressant overdose. Am J Emerg Med 11(4): 336-341.

15. Bosse GM, Barefoot JA, Pfeifer MP, Rodgers GC (1995) Comparison of three methods of gut decontamination in tricyclic antidepressant overdose. J Emerg Med 13(2): 203-209.

16. Henry JA, Alexander CA, Sener EK (1995) Relative mortality from overdose of antidepressants. BMJ 310(6974): 221-224.

17. Hollister L (1987) Antidepressants. In: Katzung BG (Eds.). Basic and clinical pharmacology ( $3^{\text {rd }}$ Edn.). Appleton and Lange, Connecticut, US, pp. 327-335.

18. Jarvis MR (1991) Clinical pharmacokinetics of tricyclic antidepressant overdose. Psychopharmacol Bull 27(4): 541-550.

19. Crome P (1986) Poisoning due to tricyclic antidepressant overdose. Clinical presentation and treatment. Med Toxicol 1(4): 261-285.

20. Spiker D, Biggs J (1976) Tricyclic antidepressants (prolonged plasma levels after overdose). JAMA 236: 1711-1712.

21. Vieweg WVR, Wood MA (2004) Tricyclic Antidepressants, QT Interval Prolongation, and Torsade de Pointes. Psychosomatics 45(5): 371-377.

22. Çitak A, Soysal Dd, Üçsel R, Karab cüoglu M, Uzel N (2006) Seizures associated with poisoning in children: tricyclic antidepressant intoxication. Pediatrics International 48(6): 582-585.

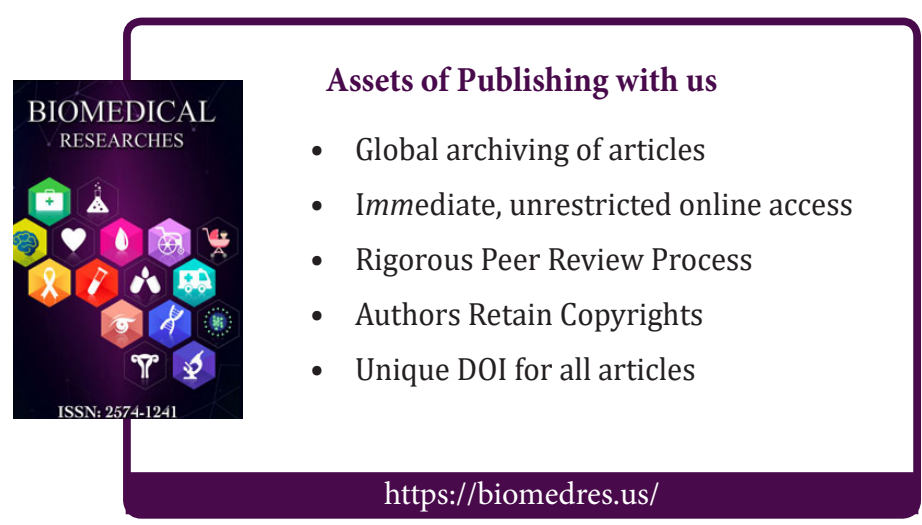

Cite this article: A Fernandez-Ibanez, R Ugalde H, JA Rodriguez G, JB Garcia-Casas, JC Diaz Suarez. Clinical Manifestations in Intoxications by Tricyclic Antidepressants. Biomed Sci\&Tech Res 8(3)- 2018. BJSTR MS.ID.001659. DOI: 10.26717/ BJSTR.2018.08.001659. 